\title{
Thermodynamic Efficiency at Maximum Power
}

\author{
C. Van den Broeck \\ Hasselt University, B-3590 Diepenbeek, Belgium \\ (Received 14 July 2005; published 2 November 2005)
}

\begin{abstract}
We show by general arguments from linear irreversible thermodynamics that for a heat engine, operating between reservoirs at temperatures $T_{0}$ and $T_{1}, T_{0} \geq T_{1}$, the efficiency at maximum power is bounded from above by $1-\sqrt{T_{1} / T_{0}}$.
\end{abstract}

PACS numbers: 05.70.Ln

Carnot efficiency is one of the cornerstones of thermodynamics. This concept was derived by Carnot from the impossibility of a perpetuum mobile of the second kind [1]. It was used by Clausius to define thermodynamic's most basic state function, namely, the entropy [2]. The Carnot cycle deals with the extraction, during one full cycle, of an amount of work $W$ from an amount of heat $Q$, flowing from a hot reservoir (temperature $T_{0}$ ) into a cold reservoir (temperature $T_{1} \leq T_{0}$ ). The efficiency $\eta$ for doing so obeys the following inequality:

$$
\eta=\frac{W}{Q} \leq 1-\frac{T_{1}}{T_{0}}
$$

The equality sign is reached for a reversible process, entailing overall zero entropy production. For practical applications, the inequality (1) has a limited significance. Indeed a reversible process, having no preferred direction in time, has to be infinitely slow. Hence, the corresponding power is zero (finite work $W$ divided by infinite time). This observation prompted Curzon and Alhborn [3] (see also [4]) to investigate the problem of efficiency at nonzero power. They consider a Carnot construction cycling in a finite time, in which the only irreversible steps are assumed to be the transfers of heat between reservoirs and the auxiliary work-performing system (the so-called endoreversible approximation). Assuming furthermore a (linear) Fourier law for the heat transfer, they derive the following efficiency at maximal power [3]:

$$
\eta=\frac{W}{Q} \leq 1-\sqrt{\frac{T_{1}}{T_{0}}} .
$$

This formula has been shown to apply, or to be at least a good approximation, in a number of other thermodynamic machines [5], including the quantum realm [6]. Furthermore, one observes in real systems efficiencies between the Curzon-Alhborn (CA) and Carnot values, in agreement with economic considerations entailing a compromise between power and efficiency. As a result, the CA paper has triggered the development of a new field of investigation, referred to as endoreversible thermodynamics, or, more generally, finite time thermodynamics [7]. Because of the elegance of the formula, the simplicity of its derivation, and the good agreement with observed efficien- cies, the CA result has also become textbook material [8]. However, the derivation is model specific and the endoreversible approximation raises the question as to the validity and generality of the bound (2). We will show below that the Curzon-Alhborn efficiency is a fundamental result that follows, without approximation, from the theory of linear irreversible thermodynamics [9].

Our starting point is a generic setup for the extraction of work from a flow of heat, see Fig. 1(a). The system performs work, $W=-F x$, against an external force $F$ (e.g., a mechanical, chemical, or electrical force) with thermodynamically conjugate variable $x$. The corresponding thermodynamic force is $X_{1}=F / T$, where $T$ is the temperature of the system. The thermodynamic flux is $J_{1}=\dot{x}$. The dot refers to the time derivative. The power (work by the system per unit time) is thus $\dot{W}=-F \dot{x}=$ $-J_{1} X_{1} T$. The work is performed under the influence of a heat flux $\dot{Q}$ leaving the hot reservoir at temperature $T_{0}$. The cold reservoir is at temperature $T_{1}$ (where $T_{0} \geq T_{1}$ ). The corresponding thermodynamic force is $X_{2}=1 / T_{1}-$ $1 / T_{0}$, and the flux is $J_{2}=\dot{Q}$. The temperature difference $T_{0}-T_{1}=\Delta T$ is assumed to be small compared to $T_{1} \approx$ $T_{0} \approx T$ so that one can also write $X_{2}=\Delta T / T^{2}$.

Linear irreversible thermodynamics is based on the assumption of local equilibrium with the following linear relationship between the fluxes and forces:

$$
J_{1}=L_{11} X_{1}+L_{12} X_{2}, \quad J_{2}=L_{21} X_{1}+L_{22} X_{2} .
$$

The positivity of the entropy production, $d_{i} S / d t=J_{1} X_{1}+$ $J_{2} X_{2} \geq 0$, implies for the Onsager coefficients $L_{i j}$ that

$$
L_{11} \geq 0 \quad L_{22} \geq 0, \quad L_{11} L_{22}-L_{12} L_{21} \geq 0 .
$$

Furthermore, the Onsager symmetry resulting from the time reversibility of the microscopic dynamics stipulates

$$
L_{12}=L_{21} \text {. }
$$

The diagonal elements $L_{11}$ and $L_{22}$ have a direct physical meaning. For $X_{2}=0$, one finds $\dot{x}=L_{11} F / T$, hence $L_{11} / T$ is the mobility of the system in response to the external force $F$. For $X_{1}=0$, we have $\dot{Q}=L_{22} \Delta T / T^{2}$, so that $L_{22} / T^{2}$ is a coefficient of thermal conductivity. The off-diagonal elements $L_{12}=L_{21}$ describe the cross coupling. These couplings have been studied in a number of 

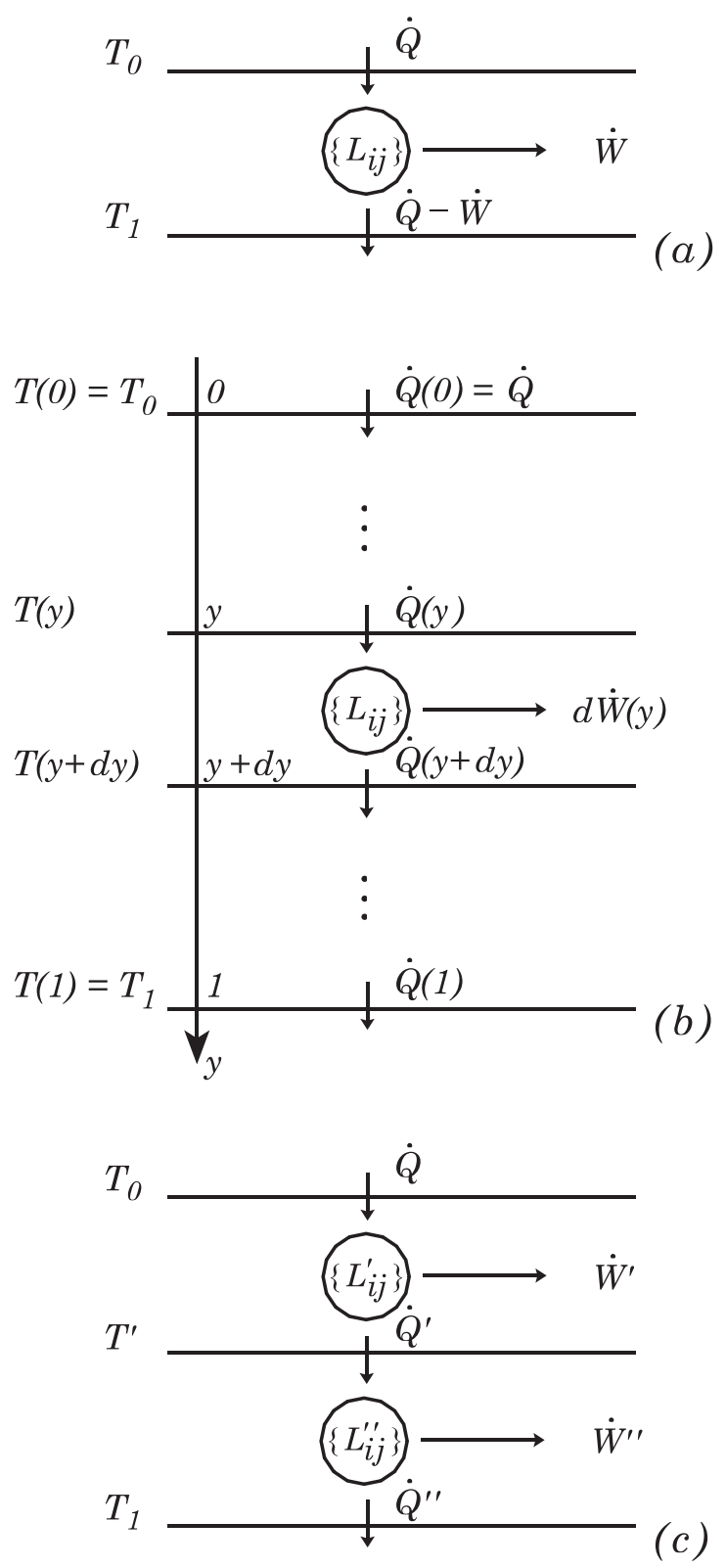

FIG. 1. (a) Generic setup for the conversion of a heat flow $\dot{Q}$ into a work flow $\dot{W}$; (b) cascade construction with a continuum of auxiliary heat baths; (c) tandem construction with one intermediate heat bath.

well-documented cases, notably the Seebeck, Thomson, and Peltier effects $[8,9]$. Note that their maximal (absolute) values are limited by Eq. (4). The dimensionless coupling strength,

$$
q=L_{12} / \sqrt{L_{11} L_{22}}
$$

thus obeys $-1 \leq q \leq+1[10]$.

After these preliminaries, the question of efficiency at maximal power can be easily addressed. One immediately concludes that the power, $\dot{W}=-J_{1} X_{1} T=-\left(L_{11} X_{1}^{2}+\right.$ $\left.L_{12} X_{1} X_{2}\right) T$, is maximal when applying a force $X_{1}^{\max }$ equal to half the stopping force, namely,

$$
X_{1}^{\max }=\frac{X_{1}^{\text {stop }}}{2} \quad \text { with } X_{1}^{\text {stop }}=-\frac{L_{12} X_{2}}{L_{11}} .
$$

The stopping force corresponds to a value of the external force $F=X_{1}^{\text {stop }} T$ for which the motion of the system halts, i.e., $J_{1}=\dot{x}=0$. The efficiency, which is the output power over input heat flux, can be conveniently written in terms of the variable $\kappa=X_{1} / X_{2}$ as follows:

$$
\eta=\frac{\dot{W}}{\dot{Q}}=-\frac{\Delta T}{T} \frac{J_{1} X_{1}}{J_{2} X_{2}}=-\frac{\Delta T}{T} \kappa \frac{L_{11} \kappa+L_{12}}{L_{21} \kappa+L_{22}} .
$$

The efficiency evaluated at maximal power, $\kappa^{\max }=$ $X_{1}^{\max } / X_{2}=-L_{12} /\left(2 L_{11}\right)$, is then found to be:

$$
\eta=\frac{1}{2} \frac{\Delta T}{T} \frac{q^{2}}{2-q^{2}}
$$

This efficiency is thus equal to half of the Carnot efficiency, $\Delta T / T$, times a factor that is a function only of the coupling strength $|q|$ defined in Eq. (6). In particular, it is independent of the overall time scale (i.e., the result is invariant upon rescaling the time). In the optimal limit of perfectly coupled systems, $|q| \rightarrow 1$, the efficiency is exactly half of the Carnot efficiency [11]. This result is valid to the lowest order in $\Delta T / T$, in which limit it actually coincides with the CA bound, Eq. (2) $\left[1-\sqrt{T_{1} / T_{0}} \sim\right.$ $\Delta T /(2 T)]$.

To go beyond the linear approximation in $\Delta T / T$, while staying within the framework of linear irreversible thermodynamics, we consider a cascade construction as in Fig. 1(b) [12]. We introduce, between the hot reservoir at $T_{0}$, and the cold one at $T_{1}$, a set of auxiliary heat reservoirs, labeled by the index $y=j / N, j=1, \ldots, N-1$ at decreasing temperatures $T(y)\left[T(0)=T_{0}, T(1)=T_{1}\right]$. These reservoirs will play a role akin to a catalyst, serving merely as temporary repositories of energy. We furthermore assume that we have at our disposal a set of $N$ identical copies of the auxiliary system, operating between the successive pairs of reservoirs. For simplicity, we assume from the onset that these machines operate at maximum power with coupling strength $|q|=1$; i.e., each of them transforms heat flux into power at half of the Carnot efficiency. Finally, we take the continuum limit $N \rightarrow \infty$. Each system and temperature reservoir is now characterized by the continuous index $y=j / N$, with the step size $d y=1 / N$ tending to zero. The heat flux traversing the reservoir located at $y$ at temperature $T(y)$, will be denoted by $\dot{Q}(y)[\dot{Q}(y=0)=\dot{Q}$ being the heat flux leaving the hot reservoir]. The incremental power delivered by the system located between $y$ and $y+d y$ is denoted by $d \dot{W}(y)$. Since the power is derived solely from the transfer of the heat, and not from the internal energy of the system, conservation of energy implies that $\dot{Q}(y+d y)=\dot{Q}(y)+d \dot{W}(y)$, whence: 
(a) $\dot{Q}(y)=\dot{Q}-\int_{0}^{y} d \dot{W}\left(y^{\prime}\right)$. Assuming that the system operates under the above-mentioned conditions (efficiency at maximum power, perfect coupling $|q|=1$ ), we have furthermore: (b) $d \dot{W}(y) / \dot{Q}(y)=[T(y)-T(y+d y)] /[2 T(y)]$. By combining (a) and (b), we obtain the following closed equation for $d \dot{W}(y) / d y$, as a function of the prescribed temperature profile:

$$
\frac{d \dot{W}(y)}{d y}=-\frac{1}{2} \frac{d \ln T(y)}{d y}\left(\dot{Q}-\int_{0}^{y} d y^{\prime} \frac{d \dot{W}\left(y^{\prime}\right)}{d y^{\prime}}\right) .
$$

Differentiating this equation with respect to $y$, one obtains a first order differential equation for $d \dot{W}(y) / d y$. Straightforward integration [with the appropriate boundary condition that follows from the application of Eq. (10) at $y=0$ ] leads to:

$$
\frac{d \dot{W}(y)}{d y}=-\frac{1}{2} \dot{Q} \sqrt{\frac{T(y)}{T(0)}} \frac{d \ln T(y)}{d y}=-\frac{\dot{Q}}{\sqrt{T(0)}} \frac{d \sqrt{T(y)}}{d y},
$$

from which the result $\dot{W}(y)=\dot{Q}[1-\sqrt{T(y) / T(0)}]$ immediately follows. We conclude that the efficiency is given by

$$
\eta=\frac{\int_{0}^{1} d \dot{W}(y)}{\dot{Q}}=\frac{\dot{W}(1)}{\dot{Q}}=1-\sqrt{\frac{T(1)}{T(0)}},
$$

which is independent of the prescribed temperature profile and precisely equal to the CA bound cf. Eq. (2).

The above analysis can be repeated for systems that are not perfectly coupled, i.e., for $|q|<1$. The dependence of the efficiency at maximum power on the ratio of temperatures is then given by $\eta\left(T_{1} / T_{0}\right)=1-\left(T_{1} / T_{0}\right)^{\rho / 2}$ with $\rho=q^{2} /\left(2-q^{2}\right)$. Hence the efficiency at maximum power is highest, namely, equal to the CA efficiency, for $\rho=1$, and deteriorates very rapidly as $|q|$ moves away from 1 . A strong cross coupling is therefore essential to be able to reach the CA bound. Interestingly, it has been conjectured that such a strong coupling is present in biochemical energy production [13].

The fact that the efficiency at maximum power is independent of the temperature profile points to an interesting invariance property, that we illustrate with a tandem construction cf. Fig. 1(c). Suppose that one system operates at CA efficiency between the high temperature heat bath (temperature $T_{0}$, heat flux $\dot{Q}$ ) and an intermediate bath at temperature $T^{\prime}$, producing a power $\dot{W}^{\prime}=\eta\left(T^{\prime} / T_{0}\right) \dot{Q}$. A second copy of the machine, working in tandem, also functions at CA efficiency between the intermediate temperature heat bath (temperature $T^{\prime}$, heat flux $\dot{Q}^{\prime}=\dot{Q}-$ $\dot{W}^{\prime}$ ) and the low temperature heat bath $T_{1}$, producing a power $\dot{W}^{\prime \prime}=\eta\left(T_{1} / T^{\prime}\right) \dot{Q}^{\prime}$. A short calculation shows that the overall efficiency is given by $\left(\dot{W}^{\prime}+\dot{W}^{\prime \prime}\right) / \dot{Q}=$ $\eta\left(T_{1} / T_{0}\right)$, i.e., the CA efficiency reproduces itself upon concatenation. This feature is also a property of machines operating at Carnot efficiency. In fact, one easily verifies that the most general function $\eta$ that reproduces this invariance (with boundary condition $\eta(1)=0$ ) is $\eta(t)=$ $1-t^{a}$. The Carnot case corresponds to $a=1$ while the CA bound corresponds to $a=1 / 2$ or more generally $a=\rho / 2$.

[1] S. Carnot, Réflexions sur la Puissance Motrice du Feu, et sur les Machines Propres à Développer cette Puissance (1824).

[2] R. Clausius, Mechanical Theory of Heat (John van Voorst, London, 1867).

[3] F. Curzon and B. Ahlborn, Am. J. Phys. 43, 22 (1975).

[4] I. I. Novikov, J. Nuclear Energy II 7, 125 (1958).

[5] H. Leff, Am. J. Phys. 55, 602 (1987); P. T. Landsberg and H. Leff, J. Phys. A 22, 4019 (1989); J. Gordon and Am. J. Phys. 57, 1136 (1989).

[6] R. Kosloff, J. Chem. Phys. 80, 1625 (1984).

[7] A. De Vos, Endoreversible Thermodynamics of Solar Energy Conversion (Oxford University, Oxford, 1992); R. S. Berry, V. A. Kazakov, S. Sieniutycz, Z. Szwast, and A. M. Tsvilin, Thermodynamic Optimization of FiniteTime Processes (John Wiley \& Sons, Chichester, 2000); P. Salamon, J. D. Nulton, G. Siragusa, T. R. Andersen, and A. Limon, Energy 26, 307 (2001).

[8] H. Callen, Thermodynamics and an Introduction to Thermostatistics (Wiley, New York, 1985), 2nd ed.

[9] I. Prigogine, Etude Thermodynamique des Phénomènes Irréversibles (Desoer, Liège, 1947); S. R. de Groot and P. Mazur, Non-Equilibrium Thermodynamics (Dover, New York, 1984).

[10] O. Kedem and S. R. Caplan, Trans. Faraday Soc. 61, 1897 (1965).

[11] This efficiency has been observed in another context: A. Bejan, Entropy Generation through Heat and Fluid Flow (John Wiley \& Sons, New York, 1982).

[12] A similar construction for endoreversible Carnot engines, is found in: M. J. Ondrechen, B. Andresen, M. Mozurkewich, and R. S. Berry, Am. J. Phys. 49, 681 (1981).

[13] J. W. Stucki, European Journal of Biochemistry 109, 269 (1980). 